\title{
Fast mixing mechanism of two vortex-current filaments
}

\author{
YUICHI YATSUYANAGI, ${ }^{1}$ TADATSUGU HATORI ${ }^{2}$ \\ and TOMOKAZU KATO ${ }^{1}$ \\ ${ }^{1}$ Department of Applied Physics, Waseda University, Shinjuku, Tokyo 169-8555, Japan \\ ${ }^{2}$ National Institute for Fusion Science, Toki, Gifu 509-5292, Japan
}

(Received 6 November 1998 and in revised from 20 July 1999)

\begin{abstract}
We demonstrate fast mixing of vortex-current filaments by means of numerical simulations of collision (strong interaction) between two straight filaments. The two filaments mutually approach, collide, and are rapidly tangled with each other. In fact, the instantaneous Lyapunov exponent shows that the dynamics becomes chaotic. Then there appear many small regions where the two filaments overlap. We consider each overlapping region to be equivalent to the traditional resistive diffusion region. We assume that the overall 'reconnection rate' of the two filaments is proportional to the product of the traditional (non-chaotic) resistive reconnection rate and the normalized overlapping volume. The overlapping volume rapidly increases on the time scale of ideal MHD. When many overlapping regions are produced, the overall reconnection probability, i.e. the sum of the probabilities of reconnection in every overlapping region, should be increased compared with that of the single overlapping region. Thus the overall reconnection rate becomes sufficiently large, although the basic reconnection process in each overlapping region is resistive and slow. We conclude that the fast mixing due to chaos may enhance the conventional resistive reconnection. We call this process 'chaotic reconnection'.
\end{abstract}

\section{Introduction}

Our investigations have been mainly motivated by the study of solar flares. Recently, much research effort has been devoted to studying solar flares because a considerable amount of observational data is available from the artificial satellite Yohkoh (Tsuneta 1997; Sterling and Hudson 1997). The energy emitted by a solar flare in one burst is in the range of $10^{20} \sim 10^{25} \mathrm{~J}$. When one considers that the total energy consumption of fossil fuels in a year in Japan is approximately $10^{19} \mathrm{~J}$, one can see how large an amount of energy is emitted by a solar flare. It would be interesting to know how such sudden and fast energy releases in solar flares are triggered.

Many researchers now share a common understanding that magnetic reconnection is a fundamental process in solar flares (Kusano et al. 1995; Fushika and Sakai 1995; Shibata 1996; Priest 1997; Sudan and Spicer 1997). For many years, electrical resistivity was considered to be a crucial mechanism in magnetic reconnection (Parker 1957; Newcomb 1958; Sweet 1958; White 1986), but it has now come to be realized that the time scale of reconnection in a solar flare cannot be explained 


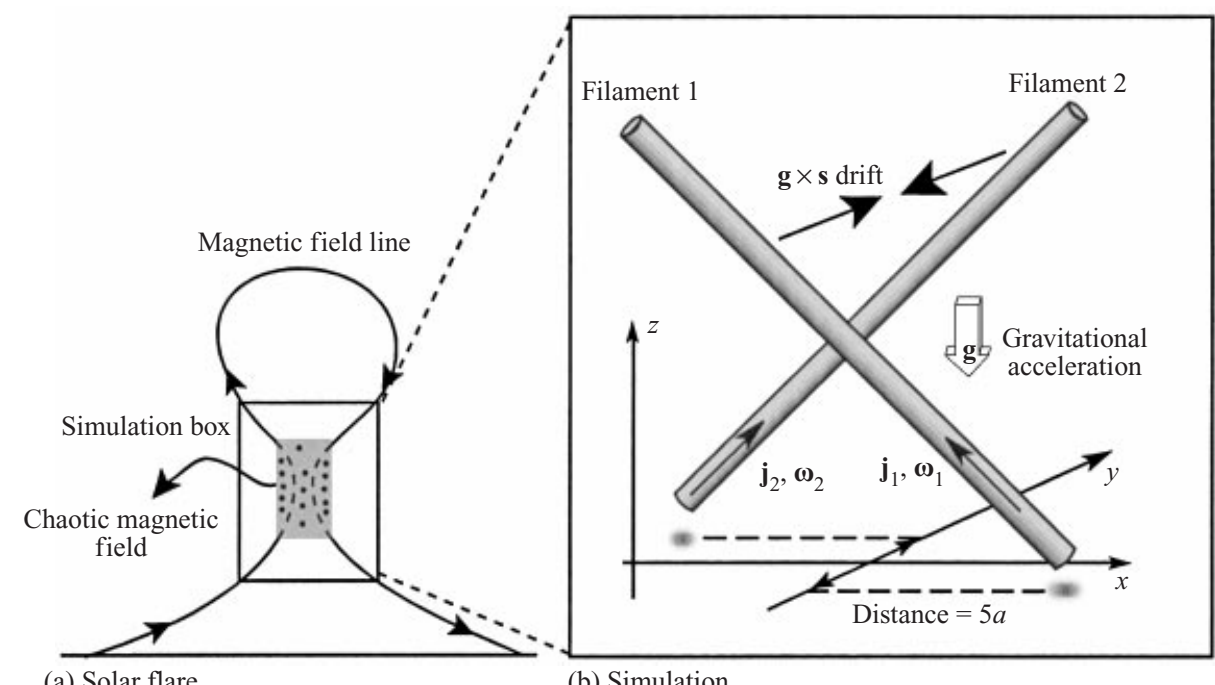

(a) Solar flare

(b) Simulation

Figure 1. (a) Schematic of a solar flare. Magnetic flux tubes are entangled and chaotic in the magnetically neutral layer, which is shown by the shaded box. The simulation region is shown by the open box. (b) Illustration of the initial condition for the simulations.

by traditional resistive reconnection theory. It is now accepted that in most cases electrical resistivity plays only a minor role, and much theoretical work on reconnection to date has dealt with the time-scale problem (Petschek 1964; Vasyliunas 1975; Hu 1983; Ichimaru 1996; Lau and Finn 1996; Tanaka 1996; Biskamp 1997; Horiuchi and Sato 1997).

We believe that the study of fast magnetic reconnection gives us a clue to clarify the mechanism of solar flares. The direct approach to analysing the fast reconnection mechanism in solar flares entails three-dimensional full magnetohydrodynamical (MHD) simulation. In our scenario, magnetic flux tubes may be entangled in the magnetically neutral layer in the solar corona, owing to the fast dynamics with high magnetic Reynolds number (see Fig. 1). It is, however, too difficult to run three-dimensional simulations because of the lack of computer resources and the high magnetic Reynolds number. As a first step, a simplified model is preferable to demonstrate the essential mechanism of magnetic reconnection due to fast mixing, and we decided to use a vortex-current filament model instead of a flux-tube model (Yatsuyanagi et al. 1996). The physical quantities to be reconnected are magnetic field lines in the traditional theory and mainly electric currents in the present work. However, we presume that essentially the same phenomenon may be demonstrated by our filament model. Note that the circulation is conserved if the vortex-current filament model is assumed. The total electric current is also conserved up to $O\left(\rho^{-2}\right)$ where $\rho$ is a local radius of curvature of the filament (Yatsuyanagi et al. 1998). This is because neither vorticity nor electric current exist outside the filament.

In this paper, we demonstrate fast mixing of the vortex-current filaments by means of 'collision' between two straight filaments. The term collision means a strong interaction between the two filaments when they mutually approach. It will be seen that the 'reconnection rate' of the filaments is enhanced by the chaotic fast mixing.

Electric current and vorticity share many features in their mathematical formu- 
lations, and much research has been done on systems in which vorticity and electric current coexist (Greene 1992; Kinney et al. 1995). On the other hand, much effort has also been devoted to studying the vortex filament and its reconnection (Moore and Saffman 1972, 1975; Fernandez et al. 1995; Zabusky et al. 1995). Motivated by the results of this work, we decided to extend the vortex filament into a vortexcurrent filament (Yatsuyanagi et al. 1996). This consists of vorticity and electric current coaxially along its axis. This model has the significant advantage that the motion of the filament is determined by very simple cutoff Biot-Savart integrals.

In our simulations, the two filaments are driven by three types of velocity: the first is a gravitational drift velocity, and the second and third are self- and mutually induced velocities by the electric current and vorticity in the filaments. Simulation results show that the filaments are tangled with each other in the collisional region where the strong interaction between them is dominant. Then their configuration is complicated because the local cylindrical symmetry of the magnetic and velocity fields induced by a filament is destroyed by the fields induced by the other filament. In the collisional region, a more asymmetrical initial configuration of the filaments yields a more complicated configuration with time.

Computations of the instantaneous Lyapunov exponents show that when the initial configuration of the filaments is asymmetrical, the exponents are positive after the collision. We find that the complicated configuration of the filaments is brought about by the chaos in the dynamical system. We call the complicated configuration 'chaotic configuration' from here on. We consider that the chaotic configuration is induced by the collision of the two vortex-current filaments, which are initially positioned asymmetrically in three-dimensional space. This is in good agreement with the results on magnetic chaos previously by one of us (Hatori and Irie 1987; Hatori et al. 1989; Urata 1990). The magnetic chaos is not observed in a two-dimensional system that is axisymmetry or has another symmetry, while it is observed in a three-dimensional system that has no symmetry. This is an important aspect of the magnetic chaos.

When the configuration of the filaments is chaotic, there appear many small regions where the filaments overlap. We consider each overlapping region to be equivalent to the conventional resistive diffusion region of magnetic reconnection. In an overlapping region, antiparallel electric currents should cancel each other. Consequently there remains a net electric current only, and the trajectory of the net electric current may have a different configuration. To obtain a new consistent trajectory of the net electric current, we introduce a three-dimensional space averaging. This is a kind of 'coarse-graining', or microscopic mixing of molecules. It is well known that coarse-graining increases the entropy of a system. This implies that coarse-graining is an irreversible process. Thus the space averaging is considered to introduce a kind of artificial dissipation process. Tracing the trajectory by the space-averaged distribution of the electric current, we obtain a reconnected configuration of the filament. If the effect of electric current on the filament motion is stronger than that of vorticity, the filaments are reconnected following the directions of the electric currents, and vice versa.

We introduce a new concept, normalized overlapping volume, to solve the timescale problem. The normalized overlapping volume is defined as the volume of the overlapping region divided by the volume of the non-chaotic one. We assume that the overall 'reconnection rate' of the filaments is proportional to the product of the traditional (non-chaotic) resistive reconnection rate and the normalized overlapping 
volume. Simulation results show that the overlapping volume rapidly increases owing to the chaotic dynamics based on ideal MHD. When many overlapping regions are produced, the overall reconnection probability, i.e. the sum of the probabilities of reconnection in every overlapping region, should be increased compared with that of a single overlapping region. Thus the reconnection rate becomes sufficiently large, although the basic reconnection process in each overlapping region is resistive and slow. We conclude that fast mixing due to chaos may enhance the conventional resistive reconnection that has a simple configuration of the magnetic field, for example the Sweet-Parker model, and no effective enhancement by the overlapping volume. We call this process 'chaotic reconnection' (Yatsuyanagi 1998).

This paper is organized as follows. In Sec. 2, we give basic equations, brief explanations of the vortex-current filament, and an approximation of the cutoff BiotSavart integral. In Sec. 3, we give results of the numerical simulations of the collision of the two filaments, and diagnoses through the instantaneous Lyapunov exponent. In Sec. 4, we explain how the filaments are reconnected through the chaotic configuration of the filament induced by the collision. Finally, in Sec. 5, we present a discussion and our conclusions.

\section{Basic equations}

We introduced the vortex-current filament model in our previous papers (Yatsuyanagi et al. 1996, 1998). The vortex-current filament consists of electric current and vorticity in the filament, and there is neither electric current nor vorticity outside. Schematics of the vortex-current filament are shown in Fig. 2. We denote a core radius and a local radius of curvature of the filament as $a$ and $\rho$, respectively. To identify a point on the filament, a position vector $\mathbf{R}$ is used. We use the natural coordinates defined by unit vectors $(\mathbf{s}, \mathbf{n}, \mathbf{b})$, where $\mathbf{s}$ is tangential, $\mathbf{n}$ is normal and $\mathbf{b}$ is binormal to the filament. We consider a small volume element $\Delta$ in the filament, with length $d s$ surrounded by a curved surface $\mathrm{C}$ and plane ends $\mathrm{E}_{1}$ at $s$ and $\mathrm{E}_{2}$ at $s+d s$. The plane ends $\mathbf{E}_{1}$ and $\mathbf{E}_{2}$ are perpendicular to $\mathbf{s}$.

As basic equations, we use the ideal MHD equations with gravitational acceleration $\mathbf{g}$ :

$$
\begin{aligned}
\frac{\partial \mathbf{u}}{\partial t}+(\mathbf{u} \cdot \boldsymbol{\nabla}) \mathbf{u} & =\mathbf{j} \times \mathbf{B}-\nabla p+\mathbf{g}, \\
\boldsymbol{\nabla} \cdot \mathbf{u} & =0 \\
\boldsymbol{\nabla} \times \mathbf{u} & =\boldsymbol{\omega} \\
\frac{\partial \mathbf{B}}{\partial t} & =-\boldsymbol{\nabla} \times \mathbf{E} \\
\nabla \cdot \mathbf{B} & =0 \\
\nabla \times \mathbf{B} & =\mu_{0} \mathbf{j} \\
\mathbf{E}+\mathbf{u} \times \mathbf{B} & =0
\end{aligned}
$$

Here the mass density is constant and normalized to unity. The scale length of the system is taken as $\hat{x}=a$, which is initially normalized to unity in the simulations. The characteristic value of the magnetic field is taken as $\hat{B}$. The Alfvén velocity and time scale are given by $\hat{u}=\hat{B} / \sqrt{\mu_{0}}$ and $\hat{t}=\hat{x} / \hat{u}$ respectively. The characteristic values of the electric current density and vorticity are denoted by $\hat{j}=\hat{B} / \hat{x} \mu_{0}$ and $\hat{\omega}=\hat{B} / \hat{x} \sqrt{\mu_{0}}$. In numerical simulations, we introduce dimensionless variables and 


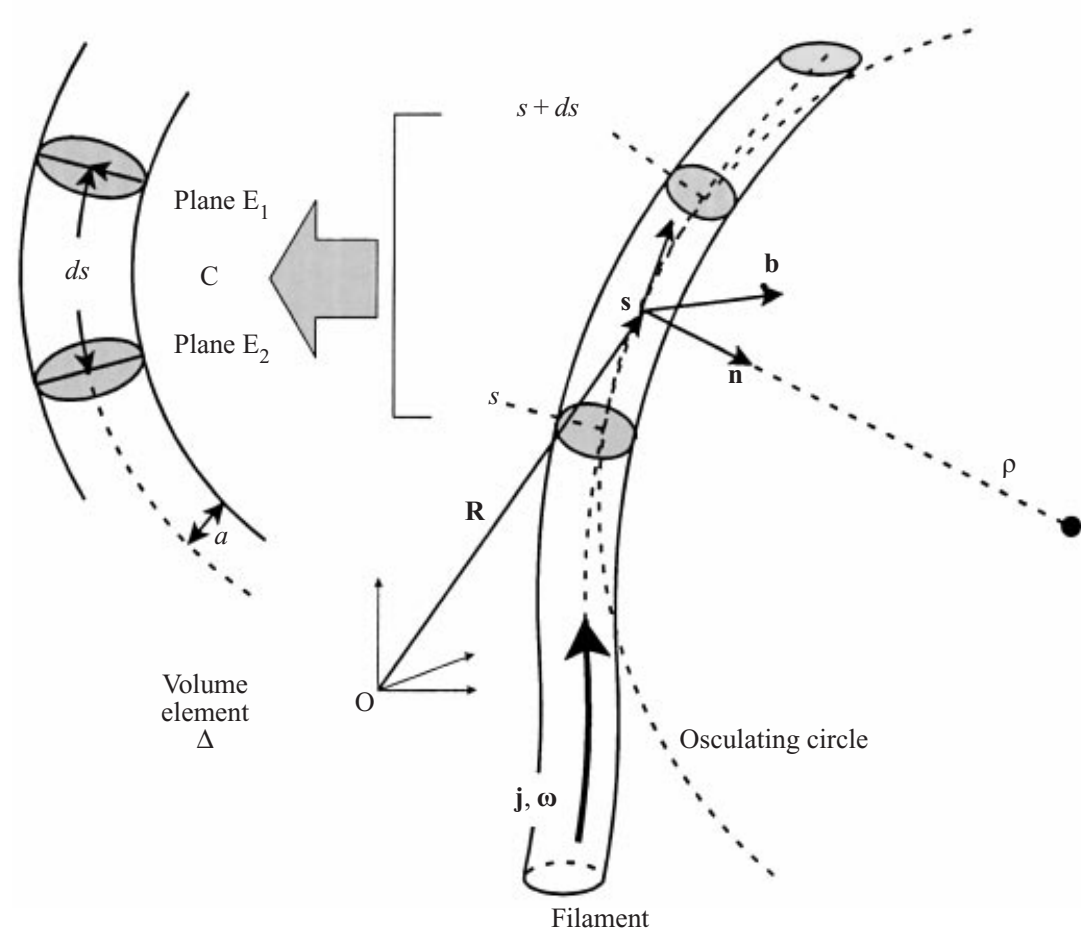

Figure 2. Schematics of the vortex-current filament and the volume element $\Delta$ of the filament, with length $d s$ surrounded by a curved surface $\mathrm{C}$ and bounded by planes $\mathrm{E}_{1}$ and $\mathrm{E}_{2}$ respectively. The core radius of the filament is $a$ and the local radius of curvature at $\mathbf{R}$ is $\rho$.

all the above-mentioned quantities are utilized as scaling factors. It is noticeable that the ratio $\hat{\omega} / \hat{j}$ has a magnitude of $\sqrt{\mu_{0}} \approx 10^{-3}$.

We now give the working formulae used in the simulations. Let us consider an interaction between filament 1 at $\mathbf{R}_{1}$ and filament 2 at $\mathbf{R}_{2}$. We use the following dimensionless equations, which give the velocities of the filaments:

$$
\begin{aligned}
\left(\frac{\partial \mathbf{R}_{1}}{\partial t}\right)_{\perp}= & \frac{J_{1}^{2}}{4 \pi \kappa_{1}} \int \frac{\left(\mathbf{R}_{1}-\mathbf{x}_{1}\right) \times \mathrm{d} \mathbf{x}_{1}}{\left(\left|\mathbf{R}_{1}-\mathbf{x}_{1}\right|^{2}+\alpha_{1}^{2} a_{1}^{2}\right)^{3 / 2}}-\frac{\kappa_{1}}{4 \pi} \int \frac{\left(\mathbf{R}_{1}-\mathbf{x}_{1}\right) \times \mathrm{d} \mathbf{x}_{1}}{\left(\left|\mathbf{R}_{1}-\mathbf{x}_{1}\right|^{2}+\beta_{1}^{2} a_{1}^{2}\right)^{3 / 2}} \\
& +\frac{J_{1} J_{2}}{4 \pi \kappa_{1}} \int \frac{\left(\mathbf{R}_{1}-\mathbf{x}_{2}\right) \times \mathrm{d} \mathbf{x}_{2}}{\left(\left|\mathbf{R}_{1}-\mathbf{x}_{2}\right|^{2}+\alpha_{2}^{2} a_{2}^{2}\right)^{3 / 2}}-\frac{\kappa_{2}}{4 \pi} \int \frac{\left(\mathbf{R}_{1}-\mathbf{x}_{2}\right) \times \mathrm{d} \mathbf{x}_{2}}{\left(\left|\mathbf{R}_{1}-\mathbf{x}_{2}\right|^{2}+\beta_{2}^{2} a_{2}^{2}\right)^{3 / 2}} \\
& +\frac{\pi a^{2}}{\kappa_{1}} \mathbf{g} \times \mathbf{s}_{1}, \\
\left(\frac{\partial \mathbf{R}_{2}}{\partial t}\right)_{\perp}= & \frac{J_{2}^{2}}{4 \pi \kappa_{2}} \int \frac{\left(\mathbf{R}_{2}-\mathbf{x}_{2}\right) \times \mathrm{d} \mathbf{x}_{2}}{\left(\left|\mathbf{R}_{2}-\mathbf{x}_{2}\right|^{2}+\alpha_{2}^{2} a_{2}^{2}\right)^{3 / 2}}-\frac{\kappa_{2}}{4 \pi} \int \frac{\left(\mathbf{R}_{2}-\mathbf{x}_{2}\right) \times \mathrm{d} \mathbf{x}_{2}}{\left(\left|\mathbf{R}_{2}-\mathbf{x}_{2}\right|^{2}+\beta_{2}^{2} a_{2}^{2}\right)^{3 / 2}} \\
& +\frac{J_{1} J_{2}}{4 \pi \kappa_{2}} \int \frac{\left(\mathbf{R}_{2}-\mathbf{x}_{1}\right) \times \mathrm{d} \mathbf{x}_{1}}{\left(\left|\mathbf{R}_{2}-\mathbf{x}_{1}\right|^{2}+\alpha_{1}^{2} a_{1}^{2}\right)^{3 / 2}}-\frac{\kappa_{1}}{4 \pi} \int \frac{\left(\mathbf{R}_{2}-\mathbf{x}_{1}\right) \times \mathrm{d} \mathbf{x}_{1}}{\left(\left|\mathbf{R}_{2}-\mathbf{x}_{1}\right|^{2}+\beta_{1}^{2} a_{1}^{2}\right)^{3 / 2}} \\
& +\frac{\pi a^{2}}{\kappa_{2}} \mathbf{g} \times \mathbf{s}_{2},
\end{aligned}
$$

where subscripts 1 and 2 indicate quantities with respect to filaments 1 and 2 
respectively. $\kappa_{n}$ and $J_{n}$ are the circulation and total electric current in filament $n$. Details are given in Appendix A.

\section{Chaotic configuration induced by the collision of two filaments}

\subsection{Collision of two filaments}

We present simulation results for the collision of two filaments. An illustration of the initial condition for the simulations is given in Fig. 1(b). The two filaments are initially parallel to the $(x, z)$ plane. The distance between the filaments is 5 scaled in the initial core radius of the filament. The gravitational acceleration is in the $-z$ direction, and we can control the mutual velocity at the collision by introducing this acceleration. The signs of the total electric current and the circulation are positive if the directions of the electric current density and the vorticity are the same as the arrows in the figure.

The most crucial parameter in our simulations is the symmetry of the initial configuration of the filaments. The symmetry of the initial configuration is determined by the initial angle $\theta$ between the two filaments projected onto the $(x, z)$ plane. The values of the initial angle are chosen as $\frac{1}{2} \pi, 2 \arctan \frac{1}{2}$ and 0 , which we shall call types (i), (ii) and (iii), respectively. The most symmetrical case is $\theta=0$ and the most asymmetrical case is $\theta=\frac{1}{2} \pi$.

The values of the total electric currents $J_{1}$ and $J_{2}$ are chosen as 2 and those of the circulations $\kappa_{1}$ and $\kappa_{2}$ as 1 . These values are constant. The initial values of $w$, $v_{0}, B_{\|}$and $B_{0}$ are chosen as $1.58,1.0,2.85$ and 0.5 respectively. These parameters are chosen such that the initial values of the cutoff parameters $\alpha$ and $\beta$ are equal to 1 . The ends of the filament are a free boundary, and we use extrapolation to satisfy the free-boundary condition. Time in the simulation is denoted by $T$, which is in multiples of $\Delta t=10^{-5}$.

We show typical results of the simulations in Figs $3-5$. We verify our simulation code; and the validity is discussed in Appendix B. Although the results in Figs 3 and 4 show complicated configurations near the collisional region, the result in Fig. 5 shows a non-complicated configuration. This is because the initial configurations for Figs 3 and 4 are more asymmetrical than that for Fig. 5. If the initial configuration is asymmetrical, the local cylindrical symmetry of the magnetic and velocity fields induced by a filament is destroyed by the fields induced by the other filament. Thus we conclude that, in the collisional region, a more asymmetrical initial configuration of the filaments yields a more complicated configuration with time.

\subsection{Diagnoses via the instantaneous Lyapunov exponents}

In the previous subsection, we have shown the complicated configuration caused by the collision of the two vortex-current filaments. The next step is to diagnose whether or not the complicated configuration in the collisional region is chaotic. To carry out the diagnosis, we use the instantaneous Lyapunov exponent. Details are given in Appendix C. In Fig. 6 we show the time development of the instantaneous Lyapunov exponent from $T=0$ to $100 \times 10^{4} \Delta t$. 

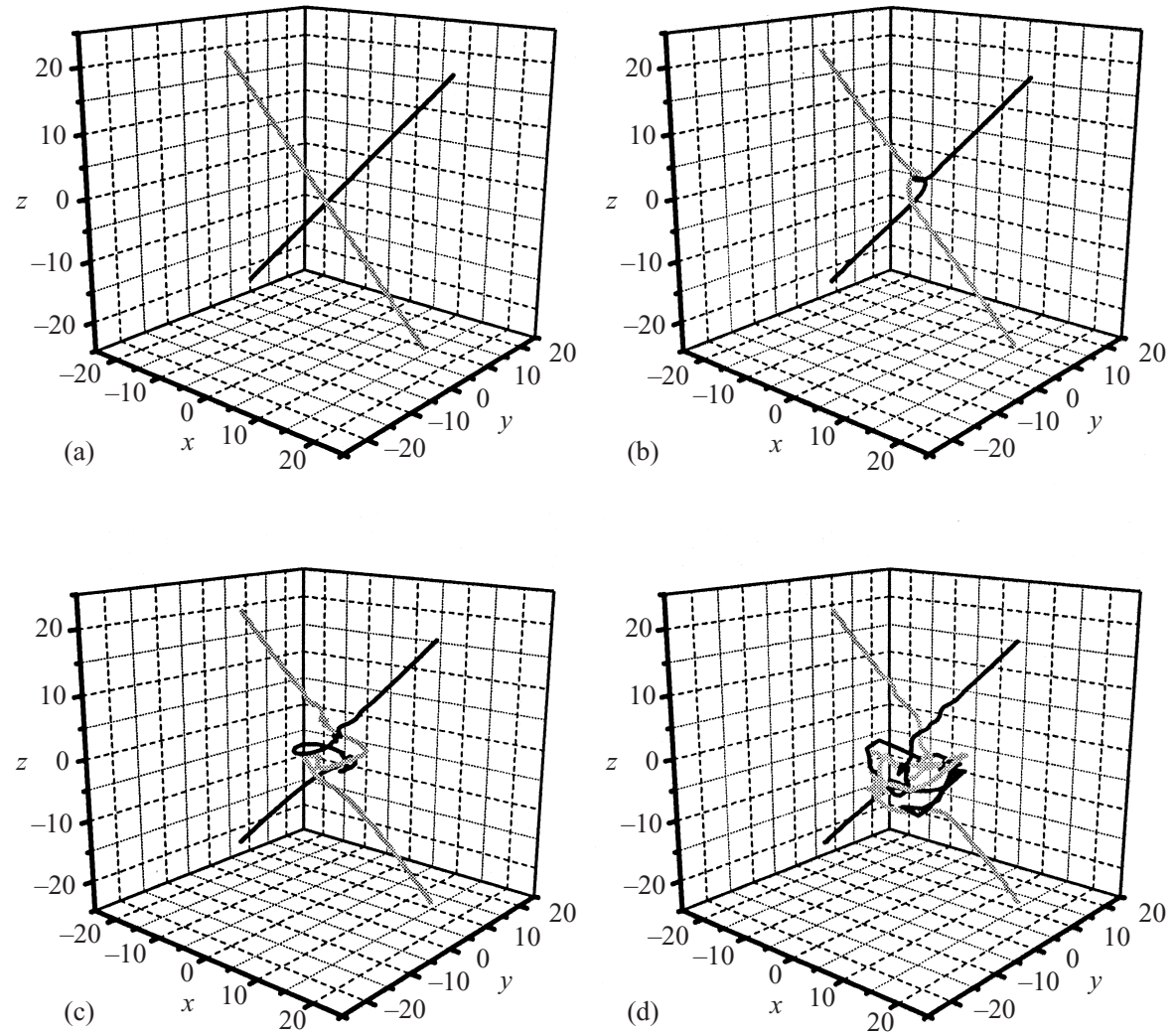

Figure 3. Time evolution of filament motion for type (i). The initial angle $\theta=\frac{1}{2} \pi$ in (a). Time develops from (a) to (d): (a) $T=0$; (b) $T=25 \times 10^{4} \Delta t$; (c) $T=50 \times 10^{4} \Delta t$; (d) $T=75 \times 10^{4} \Delta t$.

The type (iii) curve (dotted) has a spike-like peak at $T=25.5 \times 10^{4} \Delta t$. On the other hand, the two filaments in Fig. 5 are gradually attracted to each other by gravitational drift, collide and then leave in the opposite direction. The computed value of the collision time is $25.4 \times 10^{4} \Delta t$, when the mean distance between the filaments becomes zero. This time coincides with the time of the peak of the exponents for type (iii). We consider this peak to be caused by the collision of the filaments, because the two filaments are initially parallel and the collision occurs everywhere along the filament simultaneously. The peaks of the other curves at about $T=25 \times 10^{4} \Delta t$ can be explained by the same reason.

Here special attention should be paid to the values during the period from $T=50 \times 10^{4} \Delta t$ to $80 \times 10^{4} \Delta t$. The types (i) and (ii) curves show non-zero values during this period while the type (iii) curve shows near-zero values. This result shows that the instantaneous Lyapunov exponents are positive after the collision only for the initially low-symmetry configurations. That is, the collision involves the orbital instability that is observed in general chaotic dynamical systems. We therefore conclude that the chaotic configuration is induced by the collision of the two filaments with initially low-symmetry configuration. This is in good agreement with the results for magnetic chaos, which is not observed in the symmetrical system. 

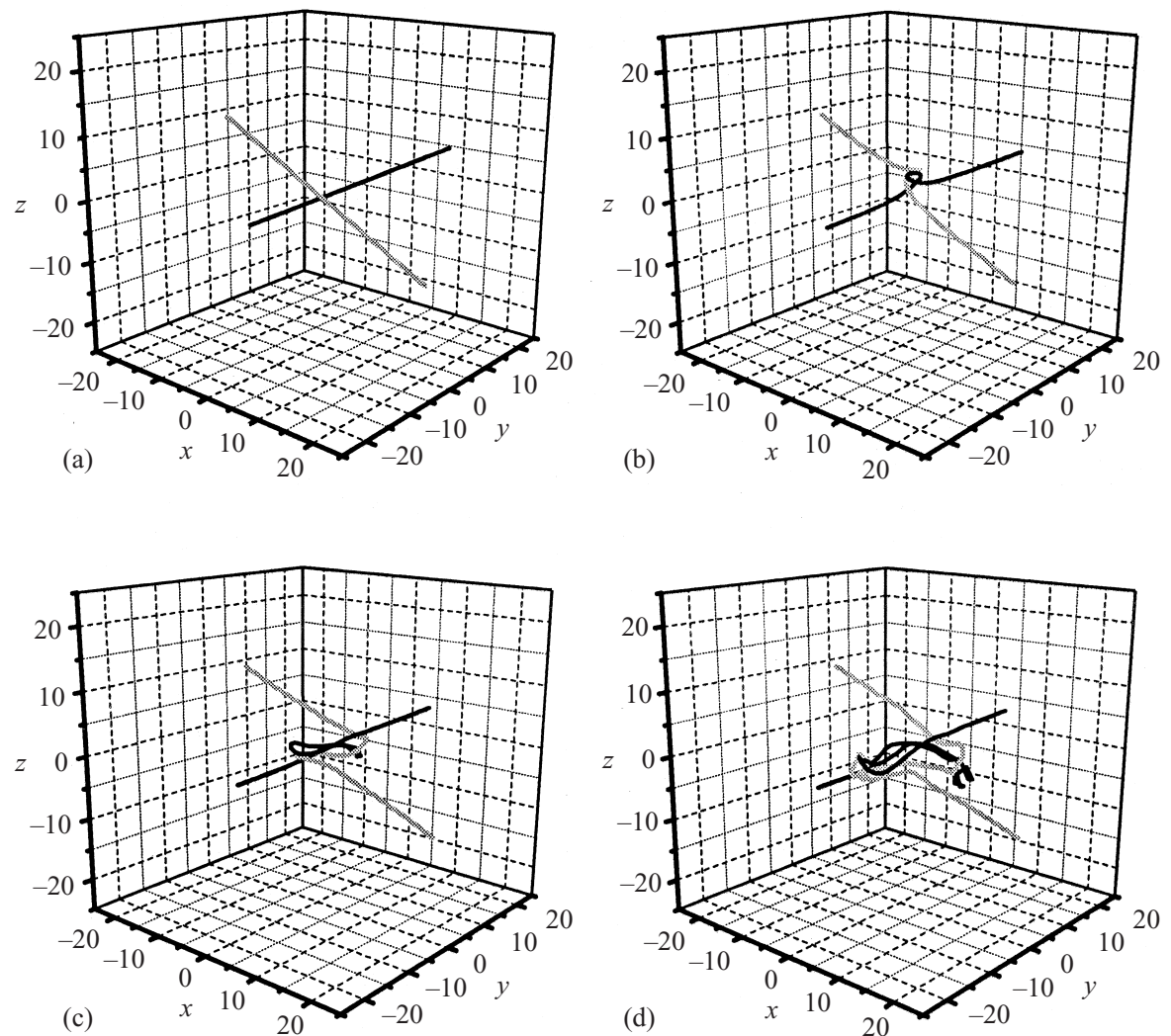

Figure 4. Time evolution of the filament motion for type (ii). The initial angle $\theta=2 \arctan \frac{1}{2}$ in (a). Time develops from (a) to (d): (a) $T=0$; (b) $T=25 \times 10^{4} \Delta t$; (c) $T=50 \times 10^{4} \Delta t$; (d) $T=75 \times 10^{4} \Delta t$.

\section{Chaotic reconnection}

The short time scale of the reconnection processes is an important issue. In this section, we propose a new fast reconnection mechanism of the filaments - a (resistive) chaotic reconnection.

In the simulations, initial volume elements inside a filament stay in the same filament eternally, even if the two filaments are tangled with each other, i.e. the position vector $\mathbf{R}_{1}$ always points to filament 1 and $\mathbf{R}_{2}$ points to filament 2 . This means that the filaments do not merge with each other in our simulations.

We do, however, consider that when filament 1 approaches filament 2 and the electric currents are antiparallel to each other, the net electric current must be nearly zero. (Of course, the magnitudes of the electric currents must be the same.) In the same manner, the vorticities are annihilated. This means that the filaments should be locally annihilated by each other in such a region. How can we resolve the inconsistency? An answer we obtain involves a three-dimensional space averaging. This is a kind of 'coarse-graining', or microscopic mixing of molecules. It is well known that coarse-graining increases the entropy of a system. This implies that the coarse-graining is an irreversible process. Thus the space averaging introduces an artificial dissipation process into the system. Note that the time scale of chaotic 

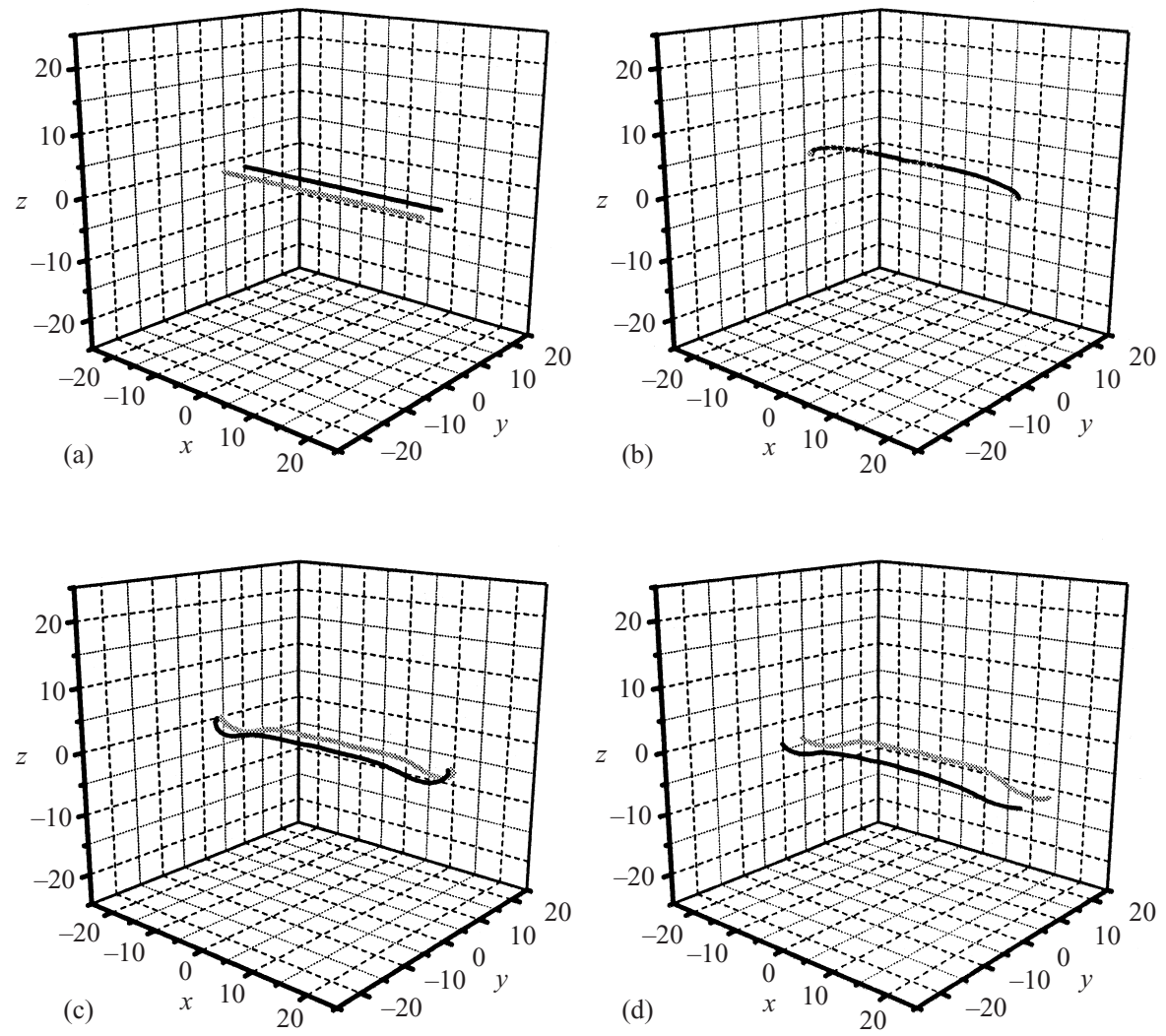

Figure 5. Time evolution of the filament motion for type (iii). The initial angle $\theta=0$ at (a). Time develops from (a) to (d): (a) $T=0$; (b) $T=25 \times 10^{4} \Delta t$; (c) $T=50 \times 10^{4} \Delta t$; (d) $T=75 \times 10^{4} \Delta t$.

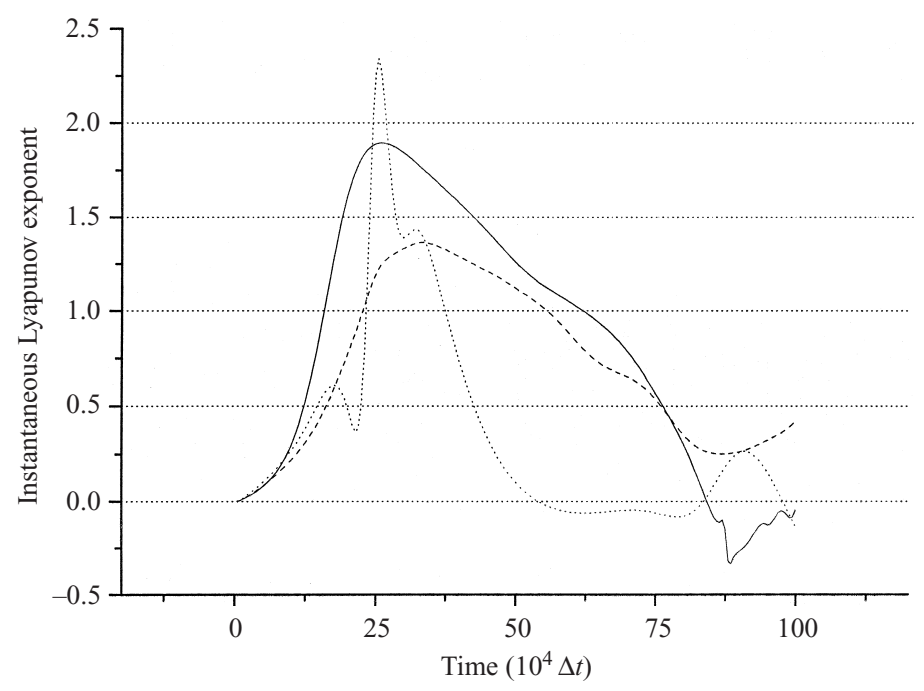

Figure 6. Time evolution of the instantaneous Lyapunov exponents from time $T=0$ to $100 \times 10^{4} \Delta t$ for type (i) (solid line), type (ii) (dashed line) and type (iii) (dotted line). 
dynamics should be determined by the Alfvén transit time and that the dissipation process introduced here does not affect the main chaotic dynamics of the filaments.

We calculate a three-dimensional space-averaged distribution of the electric currents (or vorticities) numerically, and trace the trajectory by its distribution. The procedure to obtain the trajectory is as follows:

$$
\frac{\partial \mathbf{R}}{\partial s} \propto\langle\mathbf{j}\rangle,
$$

or

$$
\frac{\partial \mathbf{R}}{\partial s} \propto\langle\boldsymbol{\omega}\rangle .
$$

where $\langle\mathbf{A}\rangle$ denotes the space-averaged distribution of $\mathbf{A}$. In the simulation region, we make cubic meshes of side $2 \alpha(t) a(t)$, which is the effective diameter of the filament. In each mesh, we calculate $\langle\mathbf{A}\rangle$. Our working formulae consist of the cutoff BiotSavart integrals. From (A 3) and (A 4), the internal structure of the filament is determined by $B_{\|}, B_{0}, w$ and $v_{0}$, and its contribution is included in the cutoff parameters $\alpha(t)$ and $\beta(t)$. So, the effective radius of the filament should be either $\alpha(t) a(t)$ or $\beta(t) a(t)$, while the material (actual) radius of the filament is $a(t)$. Now we consider a system with electric current and vorticity. In such a system, the effect of electric current is dominant. Thus we choose the effective radius of the filament to be $\alpha(t) a(t)$, which is mainly determined by the magnetic field inside the filament.

The results are shown in Figs $7(\mathrm{a}-\mathrm{c})$, in which the traced trajectories of the electric currents are shown. In Figs $7(a, b)$, the macroscopic filaments, which mean the reconstructed filaments obtained by tracing the distribution of the electric current, are reconnected with each other. The reconnection is due to the chaotic configuration induced by the collision of the two filaments in the low-symmetry system. Thus it is obvious that reconnection is not observed for type (iii) because the initial configuration is symmetrical and the configuration does not evolve into a chaotic one. We call this reconnection mechanism 'chaotic reconnection'.

One can estimate the efficiency of the reconnection process by the reconnection rate. We assume that the overall 'reconnection rate' of the filaments $R_{f}(t)$ is given by

$$
R_{f}(t)=D(t) \Omega(t, \theta),
$$

where $D(t)$ is the conventional (non-chaotic) reconnection rate determined by the resistive dissipation process of the system. $\Omega(t, \theta)$ represents the normalized overlapping volume. $D(t)$ is zero in the case of ideal MHD and positive in the case of non-ideal MHD. It is well known that a kind of dissipation process is necessary for magnetic reconnection. Thus a dissipation process, three-dimensional space averaging, has been introduced in the present paper because $D(t)$ should be finite.

With regard to fast reconnection, there has been considerable research interest in new dissipation processes, microscopic collisionless dissipation (Vekstein and Priest 1995; Priest 1997), and anomalous resistivity (Kulsrud 1998), instead of the conventional electric resistivity. Here we introduce another mesoscopic enhancement factor, normalized overlapping volume, to solve the time-scale problem for the fast reconnection. The term 'mesoscopic' means the scale that is describable by MHD but is much less than the scale length of the phenomena, while the collisionless process is microscopic. The normalized overlapping volume $\Omega(t, \theta)$ in (4.2) is introduced because the greater the number of overlapping regions, the more efficient 

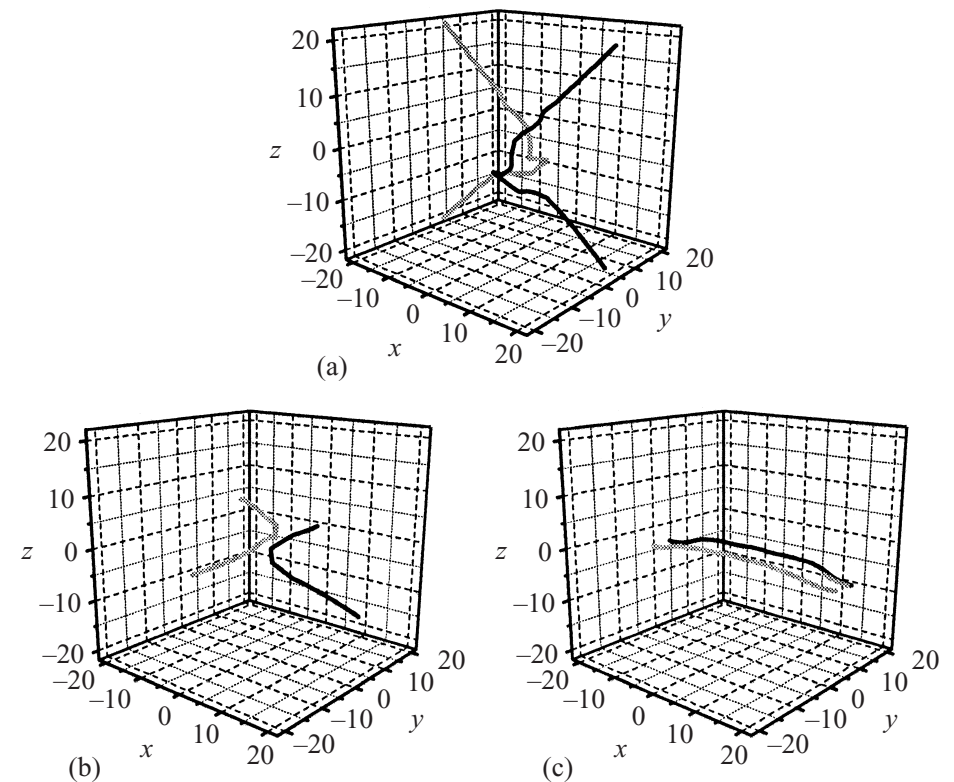

Figure 7. Trajectories of the three-dimensional space-averaged distribution of the electric current. Original data are given in Fig. 3(d) for (a), Fig. 4(d) for (b) and Fig. 5(d) for (c).

is the reconnection process evolving via the annihilation of electric currents and vorticities. Thus large $\Omega(t, \theta)$ yields fast reconnection.

The unnormalized (actual) overlapping volume is defined as the volume in which filament 1 with effective radius $\alpha_{1}(t) a_{1}(t)$ overlaps with filament 2 with effective radius $\alpha_{2}(t) a_{2}(t)$, i.e. the total volume of the overlapping regions. We must compare the resulting overlapping volumes starting from different initial configurations and investigate their magnitudes quantitatively. The magnitude for each initial configuration at the instant of collision depends on the initial angle $\theta$. We should therefore normalize their magnitudes using the non-chaotic overlapping volume derived from the initial angle. Let us consider two straight filaments that collide without magnetic or hydrodynamic interactions and mutually intrude without deformation. The overlapping volume for any initial angle is maximum when the axes of the filaments cross, provided that the radii of the filaments are the same. We adopt the maximum volume as the non-chaotic overlapping volume in the normalization and denote it by $V_{\text {nc }}(\theta)$. The normalized overlapping volume $\Omega(t, \theta)$ is defined as

$$
\Omega(t, \theta)=\frac{V_{a}(t)}{V_{n c}(\theta)},
$$

where $V_{a}(t)$ is the unnormalized overlapping volume. The non-chaotic overlapping volume is approximately proportional to $[\alpha(0) a(0)]^{3} / \sin \theta$ for types (i) and (ii) and $[\alpha(0) a(0)]^{2} L(0)$ for type (iii), where $L(0)$ is the initial length of the filament. The values of $\alpha(0), a(0)$ and $L(0)$ are 1,1 and 40 , respectively. The value of $V_{n c}(\theta)$ is 1 for type (i), 1.25 for type (ii) and 40 for type (iii). We thus obtain the normalized 




Figure 8. Time evolution of the normalized overlapping volume $\Omega(t, \theta)$ for type (i) (solid line), type (ii) (dashed line) and type (iii) (dotted line).

overlapping volume as follows:

$$
\Omega(t, \theta)= \begin{cases}V_{\mathrm{a}}(t) & \text { for type (i), } \\ 0.8 V_{\mathrm{a}}(t) & \text { for type (ii), } \\ 0.025 V_{\mathrm{a}}(t) & \text { for type (iii). }\end{cases}
$$

The time evolution of the normalized overlapping volume $\Omega(t, \theta)$ is plotted in Fig. 8. For each type, there is a peak at about $T=25 \times 10^{4} \Delta T$, and this time coincides with the time of the first peak of the instantaneous Lyapunov exponents. Thus we conclude that these peaks are due to the collision of the filaments.

At later times, the values of the normalized overlapping volume become large for types (i) and (ii) but zero for type (iii). There are many overlapping regions, i.e. the normalized overlapping volume is large, everywhere in the collisional region along the tangled filaments as a result of the chaotic dynamics based on ideal MHD. We consider each overlapping region to be equivalent to the conventional resistive diffusion region of magnetic reconnection (see Fig. 9). Then the overall reconnection probability, i.e. the sum of the probabilities of reconnection in every overlapping region, should be increased compared with that of a single overlapping region. Thus, if the factor $D(t)$ in $(4.2)$ is non-zero, the reconnection rate $R_{f}(t)$ becomes sufficiently large, although the basic reconnection process in each overlapping region is resistive and slow. We conclude that the fast mixing due to chaos may enhance the conventional resistive reconnection with a simple configuration of the magnetic field, for example the Sweet-Parker model, and no effective enhancement by the overlapping volume. This is the main mechanism of chaotic reconnection. If the normalized overlapping volume $\Omega(t, \theta)=1$ then the reconnection time scale is the same as that of conventional (non-chaotic) resistive reconnection, which is determined only by the factor $D(t)$.

We examine the non-chaotic reconnection by changing the direction of the vorticity in filament 2 . Then the directions of the gravitational drift of the two filaments are the same, and the filaments do not collide with each other. Thus the interaction 
(a)



(b)

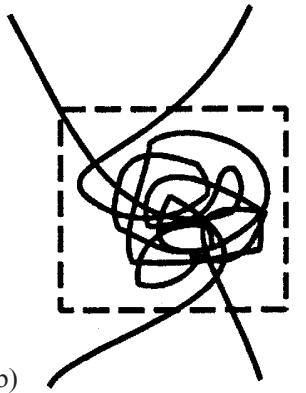

Figure 9. Illustrations of the diffusion region. (a) The diffusion region of conventional (non-chaotic) resistive reconnection. (b) The diffusion region of chaotic reconnection.

between the filaments is not as strong as the case shown in Fig. 3, and the motion is slow and non-chaotic. From computation of the instantaneous Lyapunov exponent, we find that the configuration is not chaotic. The normalized overlapping volume is also relatively small. In the case of the chaotic configuration, we can observe the reconnected configuration about 10 or more times out of 200 snapshots of the configuration, which are obtained every 5000 time-steps. In the case of the non-chaotic configuration, however, it is hard to observe the reconnected configuration. This is a good reason for us to consider that the large overlapping volume enhances the reconnection process. Thus we consider the non-chaotic reconnection to be a rare case and not to provide an efficient mechanism of fast reconnection.

Here the question arises as to which effect of electric current or vorticity is dominant in the reconnection. To answer this question, we examine a case for which the contribution of vorticity is stronger than that of electric current and the direction of the electric current in filament 2 is opposite. The result is shown in Fig. 10. The initial angle is $\frac{1}{2} \pi$. The values of the circulations are $\kappa_{1}=\kappa_{2}=5$ and the electric currents are $J_{1}=2$ and $J_{2}=-2$. In this figure, we trace the trajectories of the vorticities. We find that the filaments are reconnected following the direction of electric current or vorticity, whichever makes the larger contribution. The direction of the reconnection in Fig. 10 is reasonable because vorticity makes a larger contribution. Where, however, is the electric current? If the magnitudes of the electric currents are the same and the directions are antiparallel then the two electric currents perfectly annihilate each other and there remains no electric current (see Fig. 11). In this case, we cannot determine the detailed structure of the vortexcurrent filaments after the reconnection. We consider this to be a limitation of our vortex-current filament model.

\section{Discussion and conclusion}

The study reported in this paper may be summarized as follows. We have proposed a new fast reconnection mechanism, a resistive 'chaotic reconnection' of filaments, due to the mixing of vortex-current filaments. Note that the basic dynamics is based on nonlinear ideal MHD in which the time scale is mainly determined by the Alfvén transit time. Although we have introduced a dissipation process, i.e. three-dimensional space averaging, this does not affect the main chaotic dynamics of the filament. We have assumed the reconnection rate to be proportional to the conventional (non-chaotic) resistive reconnection rate and the overlapping volume. 

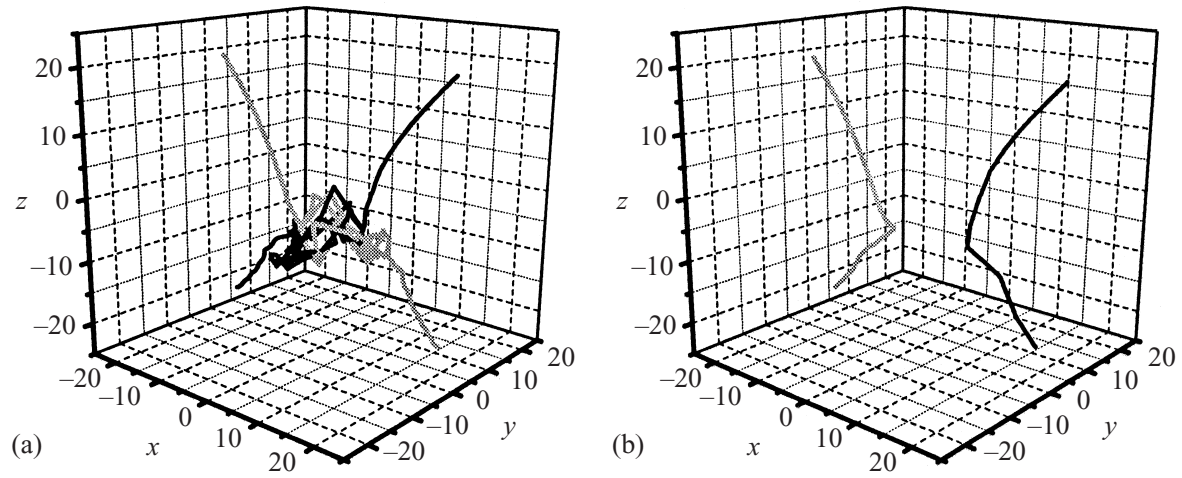

Figure 10. Trajectories of the three-dimensional space-averaged distribution of 'vorticity'. (a) The configuration of the filament at $T=75 \times 10^{4} \Delta T$. The initial angle is $\frac{1}{2} \pi$ and the values of the electric currents and the circulations are $\kappa_{1}=\kappa_{2}=5, J_{1}=2$ and $J_{2}=-2$, respectively. (b) The traced trajectory of the vorticity. The reconnected configuration is observed.


(a)


(b)

Figure 11. In which direction are the filaments reconnected and where is the electric current? The thin solid arrows show the directions of the electric currents and the dashed arrows the directions of the vorticities. In (a), the direction of the reconnection is reasonable because the directions of the electric current and vorticity inside the filament are the same. In (b), we cannot determine the detailed structure of the vortex-current filaments after the reconnection.

The simulation results show that the overlapping volume increases rapidly, owing to the chaotic dynamics based on ideal MHD. Then the reconnection rate becomes sufficiently large because the large overlapping volume enhances the reconnection process. Thus chaotic reconnection is a faster process than conventional (non-chaotic) resistive reconnection.

One of the most important physical issues concerning solar flares concerns the time scale of fast reconnection. Although the chaotic reconnection model presented in this paper is identified in using a model of the collision of two filaments and is too simplified for real solar flares, we consider that a chaotic reconnection model can be applied to fast reconnection of solar flares. In our scenario, magnetic flux tubes may be entangled in the magnetically neutral layer in the solar corona, owing 
to the fast dynamics of the high-magnetic-Reynolds-number system. The chaotic configuration of the flux tubes is considered to have a sufficiently large overlapping volume. Then a large number of diffusion regions are formed, and the probability of reconnection of the flux tubes is enhanced in the turbulent diffusion region. We must make the present model more realistic and apply it to solar flares. Further progress in understanding chaotic reconnection dynamics requires fully three-dimensional MHD simulations at high magnetic Reynolds number.

\section{Acknowledgements}

The authors gratefully acknowledge Professor K. Shibata at Kyoto University, Japan for valuable discussions. We wish to thank Dr J. M. Q. Davies for correction of errors in the English. This work was supported by a Grant-in-Aid for Encouragement of Young Scientists (Grant 10780298) from the Ministry of Education, Science, Sports and Culture, Japan, a Waseda University Grant for Special Research Project (Grant 98A-145), and Grants from the Media Network Center at Waseda University.

\section{Appendix A. Cutoff Biot-Savart integral}

Here we give some procedures to derive the working formulae $(2.2 \mathrm{a}, \mathrm{b})$ for the numerical simulations.

Integrating the equation of motion (2.1a) over the small volume element $\Delta$, we obtain a macroscopic force balance equation for the vortex-current filament correct to $O\left(\rho^{-2}\right)$. The force balance equation can be rewritten in terms of Biot-Savart integrals with cutoff parameters if the order of the equation is limited to $O\left(\rho^{-1}\right)$. The cutoff Biot-Savart integral has a positive cutoff parameter in the denominator of the integrand, and is a useful way to integrate the Biot-Savart integral numerically because the integral does not diverge, even if a source point where electric current exists coincides with a target point where a magnetic field is induced.

The following formula is the well-known result for the cutoff Biot-Savart integral that gives the velocity at a position $\mathbf{R}$ (Moore and Saffman 1972, 1975; Siggia 1985):

$$
-\frac{\kappa}{4 \pi} \int \frac{(\mathbf{R}-\mathbf{x}) \times \mathrm{d} \mathbf{x}}{\left(|\mathbf{R}-\mathbf{x}|^{2}+\beta^{2} a^{2}\right)^{3 / 2}}=\mathbf{u}_{I}-\frac{\kappa \mathbf{b}}{4 \pi \rho}\left(1-\frac{\beta^{2} a^{2}}{2 l^{2}}\right)+\frac{\kappa \mathbf{b}}{4 \pi \rho} \ln \frac{8 \rho}{\beta a}+O\left(\rho^{-2}\right),
$$

where $\beta$ is a cutoff parameter, $l$ is the length along an osculating circle of a cutoff path, $\kappa$ is the circulation of the filament, and $\mathbf{u}_{I}$ is the velocity field induced by the filament itself except in a cutoff region. Comparing our explicit version of the force balance equation with (A 1) up to $O\left(\rho^{-1}\right)$, our force balance equation is reduced to the following cutoff Biot-Savart integrals (Yatsuyanagi et al. 1996):

$$
\begin{aligned}
\left(\frac{\partial \mathbf{R}}{\partial t}\right)_{\perp}= & -\frac{J}{\kappa}\left(\mathbf{B}_{E}\right)_{\perp}+\left(\mathbf{u}_{E}\right)_{\perp} \\
& +\frac{\mu_{0} J^{2}}{4 \pi \kappa} \int \frac{(\mathbf{R}-\mathbf{x}) \times \mathrm{d} \mathbf{x}}{\left(|\mathbf{R}-\mathbf{x}|^{2}+\alpha^{2} a^{2}\right)^{3 / 2}}-\frac{\kappa}{4 \pi} \int \frac{(\mathbf{R}-\mathbf{x}) \times \mathrm{d} \mathbf{x}}{\left(|\mathbf{R}-\mathbf{x}|^{2}+\beta^{2} a^{2}\right)^{3 / 2}} \\
& +\frac{\pi a^{2}}{\kappa} \mathbf{g} \times \mathbf{s}+O\left(\rho^{-2}\right),
\end{aligned}
$$


where the cutoff parameters $\alpha$ and $\beta$ are chosen such that

$$
\begin{aligned}
-\frac{\mu_{0} J^{2}}{4 \pi \rho \kappa}\left(\ln \frac{8 \rho}{a}-\frac{1}{2}\right)-\frac{\pi a^{2}}{\rho \kappa}\left(\frac{\overline{B_{0}^{2}}}{2 \mu_{0}}-\frac{\overline{B_{\|}^{2}}}{\mu_{0}}\right) & =-\frac{\mu_{0} J^{2}}{4 \pi \rho \kappa}\left(\ln \frac{8 \rho}{\alpha a}-1\right), \\
\frac{\kappa}{4 \pi \rho}\left(\ln \frac{8 \rho}{a}-\frac{1}{2}\right)+\frac{\pi a^{2}}{\rho \kappa}\left(\frac{\overline{v_{0}^{2}}}{2}-\overline{w^{2}}\right) & =\frac{\kappa}{4 \pi \rho}\left(\ln \frac{8 \rho}{\beta a}-1\right) .
\end{aligned}
$$

The subscript $\perp$ denotes the component perpendicular to $\mathbf{s}, B_{\|}$and $w$ are the components parallel to $\mathbf{s}$ of the magnetic and velocity fields in the filament, $B_{0}$ and $v_{0}$ are the leading-order components of the azimuthal magnetic and velocity fields in the filament, and $J$ is the total electric current in the filament. $\bar{A}$ denotes the mean value of $A$ over the cross-section of the filament. The effects of the external magnetic and velocity fields are included in the terms $\mathbf{B}_{E}$ and $\mathbf{u}_{E}$ respectively. The fifth term on the right-hand side of (A 2) is the gravitational drift by which the filament is driven perpendicular to the filament axis. The working formulae $(2.2 \mathrm{a}, \mathrm{b})$ are obtained by substituting the magnetic and velocity fields induced by the other filaments for $\mathbf{B}_{E}$ and $\mathbf{u}_{E}$ into (A 2).

Note that the integral is not only introduced to avoid numerical divergence, but also derived from our force balance equation analytically. Furthermore, the velocity field determined by the cutoff Biot-Savart integral is a divergence-free field; in other words, the dynamical system is conservative (Yatsuyanagi et al. 1998).

The cutoff parameters $\alpha$ and $\beta$ are time-dependent, and are determined by three conservation laws:

$$
\begin{aligned}
L(t) a^{2}(t) & =\text { constant } \\
W(t) L(t) & =\text { constant } \\
\overline{B_{\|}}(t) a^{2}(t) & =\text { constant } .
\end{aligned}
$$

Equation (A 5) represents conservation of volume, (A 6) conservation of angular impulse or of circulation, and (A 7) conservation of magnetic flux. Details of the conservation laws are given in Yatsuyanagi et al. (1998).

\section{Appendix B. Validity check of the simulations}

In Figs 3 and 4 , the filaments at $T=75 \times 10^{4} \Delta t$ are bent strongly and become tangled with each other. On the other hand, our cutoff Biot-Savart approximation is only valid up to $O\left(\rho^{-1}\right)$. To verify our simulation results, we must test whether the local radii of curvature of the filaments are not so small as to violate the limit of the approximation. The time evolution of the percentages of mesh points where the local radius of curvature is smaller than the effective core radius of the filament $\alpha(t) a(t)$ is plotted in Fig. B.1. In this figure, the type (i) curve (solid) shows a larger rate than the other two types for most of the time, but the maximum value is not larger than $8 \%$. We conclude that our simulation results do not violate the limit of the approximation throughout the simulation time.

\section{Appendix C. Instantaneous Lyapunov exponent}

One of the most important indices used to analyse chaotic dynamical systems is the Lyapunov exponent. Recently, a great deal of research effort has been devoted 


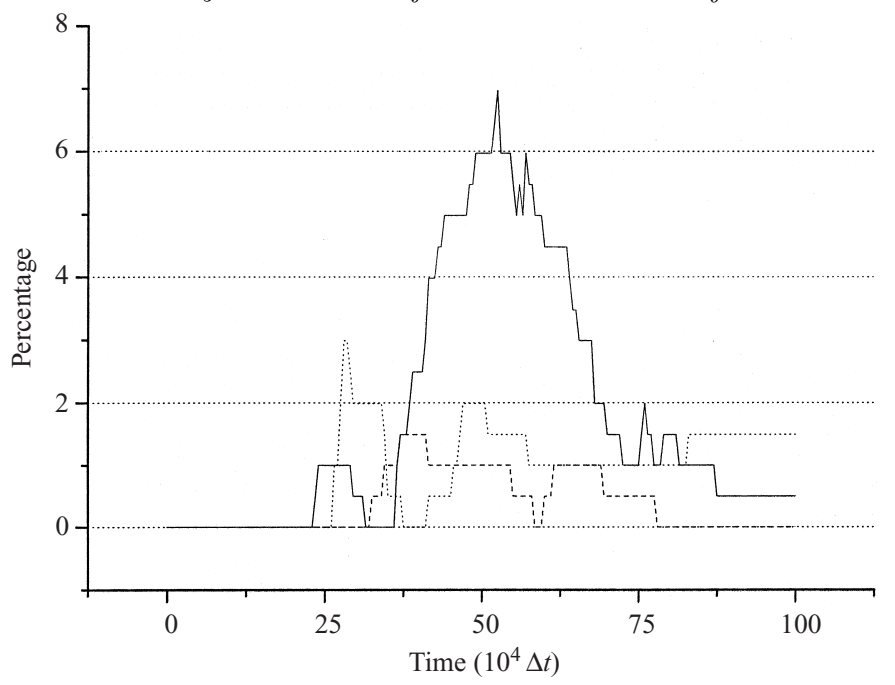

Figure B.1. The time evolution of the percentage of mesh points where the local radius of curvature is smaller than the core radius of the filament $\alpha(t) a(t)$ for type (i) (solid line), type (ii) (dashed line) and type (iii) (dotted line).

to finding the relation between Lyapunov exponents and macroscopic statistical quantities. Lyapunov exponents in phase space are dynamical characteristic quantities and are valid even in non-equilibrium situations where statistical quantities are hard to define. To analyse the complicated configuration induced by the collision of two filaments, we should calculate the Lyapunov exponents by giving a small initial displacement. In general, however, it is difficult to calculate the Lyapunov exponents numerically for a long time because of the overflow problem in the computer. To overcome this difficulty, we use a rescaling technique instead of direct calculation of the Lyapunov exponents. This provides a convenient way of calculating the instantaneous Lyapunov exponents numerically. The method is presented by Wiesel (1993) and Ueshima et al. (1997).

In our simulations of the collision of the filaments, we use 200 meshes per filament, and each mesh has three coordinates $(x, y, z)$. We consider that the edges of the filaments may be affected by numerical errors and that the most important regions for the collision are the centres of the filaments. Thus the instantaneous Lyapunov exponents are calculated using the 100 meshes at the centre of each filament. The total number of variables is 100 (meshes) $\times 3$ (coordinates) $\times 2$ (filaments $)=600$. We therefore consider a 600-dimensional phase space. From a configuration of the filaments at an instant, a point in this 600-dimensional phase space is defined. In this phase space, we calculate the instantaneous Lyapunov exponent using the rescaling method. We give an initial displacement such that filament 2 is near filament 1 by $0.01 a(0)$ in the $-y$ direction, and rescale the displacement every 5000 time-steps, rather than every one time-step.

\section{References}

Biskamp, D. 1997 Collisional and collisionless magnetic reconnection. Phys. Plasmas 4, 1964. Fernandez, V. M., Zabusky, N. J. and Gryanik, V. M. 1995 Vortex intensification and collapse of the Lissajous-elliptic ring: single- and multi-filament Biot-Savart simulations and visiometrics. J. Fluid Mech. 299, 289. 
Fushiki, T. and Sakai, J. -I. 1995 3-D simulation of plasmoid formulation during coalescence of two current loops. Sol. Phys. 156, 265.

Greene, J. M. 1992 Reconnection of vorticity lines and magnetic lines. Phys. Fluids B37, 2355

Hatori, T. and Irie, H. 1987 Long-time correlation of the chaotic orbit in the two-wave Hamiltonian. Prog. Theor. Phys. 78, 249.

Hatori, T., Irie, H., Abe, Y. and Urata, K. 1989 Chaotic magnetic field line in toroidal plasmas. Prog. Theor. Phys. Suppl. 98, 83.

Horiuchi, R. and Sato, T. 1997 Particle simulation study of collisionless driven reconnection in a sheared magnetic field. Phys. Plasmas 4, 277.

Hu, P. N. 1983 Fast reconnection of magnetic fields in a plasma. Phys. Fluids $\mathbf{2 6}, 2234$.

Ichimaru, S. 1996 Reconnection rate of magnetic-field lines in hydromagnetic plasma turbulence. Phys. Plasmas 3, 233.

Kinney, R., McWilliams, J. C. and Tajima, T. 1995 Coherent structures and turbulent cascades in two-dimensional incompressible magnetohydrodynamic turblence. Phys. Plasmas 2, 3623

Kulsrud, R. M. 1998 Magnetic reconnection in a magnetohydrodynamic plasma. Phys. Plasmas 5, 1599

Kusano, K., Suzuki, Y. and Nishikawa, K. 1995 A solar flare triggering mechanism based on the Woltjer-Taylor minimum energy principle. Astrophys. J. 441, 942.

Lau, Y. -T. and Finn, J. M. 1996 Magnetic reconnection and topology of interacting twisted flux tubes. Phys. Plasmas 3, 3983.

Moore, D. W. and Saffman, P. G. 1972 The motion of a vortex filament with axial flow. Phil. Trans. R. Soc. Lond. A272, 403.

Moore, D. W. and Saffman, P. G. 1975 The instability of a straight vortex filament in a strain field. Proc. R. Soc. Lond. A346, 413.

Newcomb, W. A. 1958 Motion of magnetic lines of force. Ann. Phys. (NY) 3, 347.

Parker, E. N. 1957 Sweet's mechanism for merging magnetic fields in conducting fluids. J. Geophys. Res. 62, 509.

Petschek, H. E. 1964 Magnetic field annihilation. In: Proceedings of AAS-NASA Symposium on the Physics of Solar Flares. NASA Spec. Publ. SP-50, p. 425.

Priest, E. R. 1997 Three-dimensional magnetic reconnection in the solar corona. Phys. Plasmas 4, 1945 .

Shibata, K. 1996 New observational facts about solar flares from Yohkoh studies. Adv. Space Res. 17, 9

Siggia, E. D. 1985 Collapse and amplification of a vortex filament. Phys. Fluids 28, 794.

Sterling, A.C. and Hudson, H.S. 1997 Yohkoh observations of X-ray 'dimming' associated with a halo coronal mass ejection. Astrophys. J. Lett. 491, 55.

Sudan, R. N. and Spicer, D. S. 1997 Conventional solar flare theory re-examined. Phys. Plasmas 4, 1929

Sweet, P. A. 1958 The neutral point theory of solar flares. In: Electromagnetic Phenomena in Cosmical Physics (ed. B. Lehnert), p. 123. Cambridge University Press.

Tanaka, M. 1996 Asymmetry and thermal effects due to parallel motion of electrons in collisionless magnetic reconnection. Phys. Plasmas 3, 4010.

Tsuneta, S. 1997 Moving plasmoid and formation of the neutral sheet in a solar flare. Astrophys. J. 483, 507.

Ueshima, Y., Nishihara, K., Barnett, D. M., Tajima, T. and Furukawa, H. 1997 Particle simulation of Lyapunov exponents in one-component strongly coupled plasmas. Phys. Rev. E55, 3439 .

Urata, K. 1990 Nonlinear coupling of the resistive tearing modes under the unperturbed shear flow. J. Phys. Soc. Jpn 59, 2742.

Vasyliunas, V. M. 1975 Theoretical models of magnetic field line merging, 1. Rev. Geophys. Space Phys. 13, 303.

Vekstein, G. E. and Priest, E. R. 1995 Nonlinear magnetic reconnection with collisionless dissipation. Phys. Plasmas $2,3169$.

White, R. B. 1986 Resistive reconnection. Rev.Mod.Phys. 58, 183. 
Wiesel, W. E. 1993 Continuous time algorithm for Lyapunov exponents. I. Phys. Rev. E47 3686 .

Yatsuyanagi, Y. 1998 Nonlinear behavior and chaotic reconnection of the vortex-current filaments in gravity field. PhD Thesis, Waseda University, Tokyo.

Yatsuyanagi, Y., Hatori, T. and Kato, T. 1996 The equations of motion of vortex-current filaments. J. Phys. Soc. Jpn 65, 745.

Yatsuyanagi, Y., Hatori, T. and Kato, T. 1998 Numerical simulations of the vortex-current filaments motion. J. Phys. Soc. Jpn 67, 166.

Zabusky, N. J., Fernandez, V. M. and Silver, D. 1995 Collapse, intensification and reconnection in vortex dominated flows. Physica D86, 1. 